\title{
Stock Price Forecasting: Review and Experiment on Time Series Data Processing
}

\author{
Jonathan $^{1}$, Andreas Pangestu Lim ${ }^{2}$, Dionisius Saviordo Thenuardi ${ }^{3}$, Gede Putra Kusuma ${ }^{4}$, Diaz D. Santika ${ }^{5}$ \\ ${ }^{1}$ Computer Science Department, BINUS Graduate Program - Master of Computer Science, \\ Bina Nusantara University, Jakarta, Indonesia, 11480, jonathan016@binus.ac.id \\ ${ }^{2}$ Computer Science Department, BINUS Graduate Program - Master of Computer Science, \\ Bina Nusantara University, Jakarta, Indonesia, 11480, andreas.lim@ @inus.ac.id \\ ${ }^{3}$ Computer Science Department, BINUS Graduate Program - Master of Computer Science, \\ Bina Nusantara University, Jakarta, Indonesia, 11480, dionisius.thenuardi@ binus.ac.id \\ ${ }^{4}$ Computer Science Department, BINUS Graduate Program - Master of Computer Science, \\ Bina Nusantara University, Jakarta, Indonesia, 11480, inegara@ binus.edu \\ ${ }^{5}$ Computer Science Department, BINUS Graduate Program - Master of Computer Science, \\ Bina Nusantara University, Jakarta, Indonesia, 11480, ddsantika@ binus.ac.id
}

\begin{abstract}
Stock price forecasting may present a huge benefit for economy sector as the ability to predict future stock prices may help related parties prepare for the predicted or forecasted condition. Many artificial intelligence-related approaches have been proposed, and this paper provides reviews of time series processing in general and stock price forecasting progresses for newcomers to get acquainted with. In addition, experimentation of stock price forecasting on several existing approaches from time series processing in general are provided to gain insight on which approach may perform better and which may merit further research on stock price forecasting task.
\end{abstract}

Key words: Stock Price Forecasting, Time Series Data, Data Mining, Review and Experiment, CNN-LSTM.

\section{INTRODUCTION}

Time series processing provides insights on what to expect in the future given series of data bound by time. This may be beneficial in many sectors of life, with one among many affected sectors being economy. Many economy aspects have been used in time series processing researches, with one most forecasted economy aspect being stock price data. Stock price forecasting may benefit in times of global pandemic such as the recent COVID-19 virus or catastrophic events that affect stock prices by allowing related parties to sell/buy stocks based on the forecast results.

Forecasting stock price has been a topic of interest in time series processing researches, reaching back to 1975 of its early publications [1]. To understand how far stock price forecasting approaches have gotten, it is imperative to understand what and how to perceive and process time series data. This paper provides a concise yet meaningful review of important progresses on time series processing in general. It is continued with a review on stock price forecasting, which include available datasets, related works and their respective results, and a summary of the related works' progresses. This review benefits readers by equipping readers with knowledge of the current state and previous attempts in time series processing and stock price forecasting, hence allowing newcomers to get familiar with what had been done and what can be improved in future researches.

Several similar researches have been done in the past, where one of them tackled the matter using Data Mining and Genetic Algorithm (GA) approaches [2]. Another research, recently done in 2020, used a tech based soft computing approach [3] to predict stock prices. This paper experimented on stock price forecasting by comparing several models to provide initial implementation ideas for newcomers and to empirically gain insight on how time series processing works, especially in stock price forecasting. On evaluating several models on local bank stock price (BBRI.JK) dataset, it was found that Convolutional Neural Network - Long Short-Term Memory (CNN-LSTM) provides the best result out of all models with Root Mean Square Error (RMSE) score of 1.129830672 .

In summary, the contributions of this paper are as follow:

1. Providing review of important milestones in time series processing in general and stock price forecasting using time series processing for general audience or newcomers to this field of study.

2. Experimentation on several models for stock price forecasting for finding out empirically which method may be of interest in future researches on stock price forecasting. 
This paper continues by providing a review on time series processing in general on Section 2. More specific details on stock price forecasting as time series problem are discussed on Section 3. Section 4 describes the conducted experiments and continued in Section 5 with the obtained results and discussion of the results. Finally, this paper concludes in Section 6 with conclusion of the review, experiments, and recommendations on stock price forecasting researches.

\section{A REVIEW ON TIME SERIES PROCESSING 2.1 The Concepts of Time Series Data}

Time series data is a data obtained of sequential data and stored in specific time atomicity, such as entry per hour or per day. This is different from other datasets, such as image recognition datasets, in that each data point in time series data relates to one another, be that directly or indirectly. While image datasets have patterns of pixel values for each category, time series datasets have patterns of each data point, and future values are usually forecasted by the patterns of the data.

In forecasting time series data points, two forecasting categories are commonly discussed: multivariate and univariate. The main difference between the former and the latter is how the pattern are formed, in that univariate time series data has only one factor, which is the data points themselves, that contribute to how the data pattern is represented, while multivariate category has more than one factor that affects how the data pattern is shown and therefore could be described as an extension of univariate category [4].

While multivariate time series data may provide better insight as to how the data pattern is formed [4], it can also be quite challenging to process as the processing pipeline must consider other factors and also how much impact each factor has over the data pattern [5]. Univariate time series data, on the other hand, is much simpler to process but may omit several key factors that contribute to how the data points' values move.

\subsection{Sample Datasets}

Time series datasets vary in terms of availability and field of study usage, which covers economy, social, medical, and spanning across other categories. Several sample datasets belonging to economy category are Dow Jones index stock prices [6] and retail transactions [7]. Social datasets such as birth rate [8] and room occupancy based on sensory data [9] are available as well. Medical datasets, namely epileptic seizure [10] to the recent cases of COVID-19 virus [11] are available for better forecasting of medical conditions in the future. These datasets vary in terms of data atomicity and their respective categories. More datasets can be found in [12-15], among many other sources available on the Internet.

\subsection{Proposed Approaches/Models}

The first attempt for processing time series data, which is a serial data, began with Recurrent Neural Network (RNN). Both Elman [16] and Jordan [17] proposed an approach for dealing with serial data, with the difference being Elman proposed the use of hidden layer as another input for the hidden layer in the next iteration, while Jordan proposed the output of output layer to be used as another input for the hidden layer in the next iteration as well.

After RNN, among the most popular proposed methods for time series processing is Long Short-Term Memory (LSTM) [18], which addresses previous approaches of learning capability. The backpropagation mechanism for models dealing with serial data at that time, including RNN, caused several problems that resulted in the model's learning process to take a very long time. In addition, RNN is not capable of dealing with serial data that spans for a long period. LSTM provides a mechanism for dealing with such problem by introducing a memory cell to retain longer information and gates for controlling the cell's information.

Bidirectional LSTM (BLSTM) [19] was proposed as a refinement for LSTM, with the benefit of being able to retain information of previous data and next data given currently processed data in training, as the training process passes previous data and the next data point as inputs. This is different from LSTM in that LSTM only retains information from previous data, and by doing this, BLSTM performs significantly better than RNN and LSTM as it is more capable of understanding the context due to the bidirectional inputs it receives.

In 2012, CNN rose to fame with AlexNet [20] due to its capability in image processing tasks by independently learning and extracting features using convolutional layers. AlexNet works by having several convolutional layers for feature extraction purposes and the extracted features are used by the fully connected layers at the end of the last convolutional layer for classification. The convolutional layers work by convoluting each pixel in the input image with a kernel. More CNN-based models were proposed afterwards as CNN made its way to other fields of study.

CNN made its way to time series processing after it was combined with LSTM [21] and known as CNN-LSTM. It was proposed to address visual-based time series data, such as sequence of images with the main purpose being to generate textual description given such time series data. CNN works as the feature extractor of the model and continued by LSTM then fully connected layers for forecasting and prediction purposes. 


\section{STOCK PRICE FORECASTING AS A TIME SERIES PROBLEM}

\subsection{Available Datasets}

One sample available dataset is the Istanbul Stock Exchange dataset [22], collected from several websites and contains 536 rows from January $5^{\text {th }}, 2009$ to February $22^{\text {nd }}, 2011$. Each row contains two Istanbul Stock Exchange values of Turkish Lira and US Dollar, stock market return index of four countries, and other index values. The dataset's atomicity is entry per day. However, several days are missing from the record.

A website [23] of huge amount of stock data of various companies is also accessible, showing daily-updated stock records covering NYSE, NASDAQ, AMEX, and ARCA indexes. Some of the companies included are The Walt Disney Company, Microsoft Corporation, The Boeing Company, and Nike Inc. However, membership to the website must be owned prior to accessing the data.

Yahoo Finance [24] also provides historical data of stock prices of varying listings and companies. Search for a company or index, and Yahoo Finance will provide the information of current value, chart of past data, historical data, and other informative data of the company or index. However, several days are also missing from the record.

\subsection{LSTM for Stock Price Forecasting}

LSTM is used to forecast NIFTY 50 stock market in [25]. Data discretization, normalization, cleaning, and integration were used for preprocessing the raw data. Several features were chosen as inputs for 2 LSTM layers with ReLU and Linear as activation function, respectively. The model achieved 0.00859 using RMSE after training for 500 epochs with $90 \%$ training and $10 \%$ testing data.

An ensemble learning approach combining LSTM and AdaBoost was proposed to integrate a set of LSTM predictors [26]. The idea of boosting algorithm is to train a set of models sequentially where each model learns from mistakes made by predecessor models. After obtaining all LSTM predictors, the final forecasting model will be generated by integrating all of LSTM predictors with ensemble weights. With ensemble of 10 forecasters, on Standard \& Poor's 500 (S\&P500) dataset, AdaBoost-LSTM outperformed other boosted methods by achieving 0.413 Mean Absolute Percentage Error (MAPE) compared to AdaBoost-MLP with 1.023, AdaBoost-SVR with 0.841, and AdaBoost-ELM with 0.782. On SHCI dataset, AdaBoost-LSTM also proved to be superior with 0.312 MAPE as opposed to AdaBoost-MLP with 0.918, AdaBoost-SVR with 1.106, and AdaBoost-ELM with 0.692 .
A multivariate time series dataset of stock price was used by including text taken from an article about a certain company [27]. The text is converted into vectors using distributed memory model of paragraph vectors (PV-DM) and distributed bag of words version of paragraph vector (PV-DBOW) before being merged. Then, the vector is fed to LSTM, trained using Adam optimizer for 50 epochs. Multiple companies with the same industry were chosen as datasets to learn the correlations between companies. The result shows that using text as additional features increase the profits made by the model, and that using multiple companies of the same industry is more effective than only one company or multiple companies from different industry.

\subsection{BLSTM for Stock Price Forecasting}

Google's stock price data was used in an experiment using BLSTM in comparison with traditional LSTM [28]. The training mechanism ran for 500 epochs with Adam optimizer and learning rate of 0.01 . The obtained result shows that BLSTM gave slightly better performance to traditional LSTM with lower minimum RMSE of 0.015, whereas traditional LSTM obtained 0.0209 RMSE score. It was also reported that the accuracy of BLSTM is $84 \%$, only $1 \%$ higher than traditional LSTM.

Another research was done utilizing BLSTM in comparison to Auto Regressive Integrated Moving Average (ARIMA) and LSTM for GREE stock price forecasting [29]. The input data was preprocessed first using normalization and standardization, and the neural network used dropout to prevent overfitting. The training process went for 100 epochs with Adam optimizer and 50 hidden layer units, and the used dataset was the GREE stock price dataset from January $1^{\text {st }}$, 2017 to May $14^{\text {th }}, 2019$ with 568 entries, with 304 entries as training set and 204 entries for test set. The lowest obtained RMSE is 1.3382 , which beats LSTM with 1.3824 RMSE score for the network without dropout.

Using S\&P500 obtained from Yahoo Finance from January $1^{\text {st }}, 2010$ to November $30^{\text {th }}, 2017$, BLSTM proved to be superior against LSTM [30]. The dataset was split for $80 \%$ training and $20 \%$ testing set, and the data was normalized in data preprocessing stage. BLSTM was evaluated for short-term and long-term forecasting, with the short-term period being one day in the future and the long-term period 30 days in the future. For both networks with 32 hidden layer units, BLSTM obtained lowest RMSE of 0.021747 on short-term forecasting compared to LSTM with RMSE of 0.025862, while for long-term forecasting, the best BLSTM's RMSE is 0.062648 while LSTM obtained RMSE score of 0.080033 . 


\subsection{CNN for Stock Price Forecasting}

CNN is usually used to process datasets with $2 \mathrm{D}$ structures instead of 1D structures, while every time series datasets are in the form of 1D structures. During the preprocessing stage, researchers would have to either transform the 1D dataset into $2 \mathrm{D}$ or the opposite. Stock exchange rate can be converted into 2D structure in the form of "time-axis x time-axis".

A team of researchers conducted an experiment to predict intraday direction of Borsa Istanbul by using CNN [31]. The dataset used in their experiment consists of hourly stock prices of 100 companies listed in BIST 100 Index ranging from January 2011 until December 2015 with $80 \%$ of the data used as training set and $20 \%$ as testing set. Two experiments were reported where the first one used CNN classifier, whereas the second one used F-Measure classifier. It was reported that their proposed $\mathrm{CNN}$ achieved better results than F-Measure using filter size of 8 , max-pooling with $2 \times 2$ size, Adadelta optimizer, ReLU activation function, 200 epochs, batch size of 32, and dropout rate of 0.3. The evaluation was done using Macro-Averaged (MA) F-Measure, and their proposed method achieved a mean rate of 0.563 which outperforms Naïve and Random methods.

Hoseinzade and Haratizadeh proposed their own kind of CNN named CNNPred [32], which is a CNN-based framework for stock market prediction. Two variations were proposed, 2D-CNNpred and 3D-CNNpred, each with their own approaches in constructing prediction models but with the same parameters for the CNN which used 8x8x8 filter size, ReLU-Sigmoid activation function, Adam optimizer, dropout rate of 0.1 , and batch size of 128 . The two variations were tested on S\&P 500, NASDAQ, Dow Jones Industrial Average, NYSE and RUSSELL market with 82 features representing each day of each market. They used MA F-Measure to evaluate their models and found that both variations had $3-11 \%$ improvement in performance than the baseline state-of-the-art algorithms.

Another research regarding stock prediction was done by Chen and He, who used CNN as the model to predict China's Stock Exchange [33]. The dataset itself was taken from January $5^{\text {th }}, 2015$ to December $29^{\text {th }}, 2017$ and then split into $80 \%$ for training and $20 \%$ for testing. Preprocessing was done by taking several features from the dataset, which are open, close, high, low, and volume. Also, removal of data with missing values and repeating data was done. The proposed model architecture consists of 2 convolutional layers, 1 pooling layer of $2 \times 2$ size and 2 fully connected layers, with the learning rate of 0.0001 and batch size of 1,000 samples. Gaussian distribution was used for weight initialization and biases' values are set to 0 . To avoid gradient vanishing, they used ReLU for the first activation function and LReLu for the rest. They achieved $75.2 \%$ accuracy in 500,000 iterations as the best accuracy.

\subsection{CNN-LSTM for Stock Price Forecasting}

CNN-LSTM has also been utilized in stock price forecasting with combination of attention mechanism, a form of summation with weights, for improving regression capability of the CNN-LSTM model and yielded good results on two evaluation datasets [34]. The used datasets are JQData which the authors collected and Pingan Bank; each contains data from April $1^{\text {st }}, 2017$ until March 30 $0^{\text {th }}, 2019$ and each was split for $90 \%$ training and $10 \%$ testing data. Input data were normalized using Z-score technique and the proposed method beat LSTM, Support Vector Regression (SVR), and Gated Recurrent Unit (GRU) on both datasets. The best MSE score was obtained on input sequence length 30, which are 0.0012 and 0.0014 for JQData and Pingan Bank datasets, respectively.

Another use of CNN-LSTM for stock forecasting is reported in [35]. The CNN-LSTM was used to understand the trend of the data, while other factors such as seasonal and event components were approximated by Fourier transformations and a linear function. The research utilized multivariate dataset of Verizon and T-mobile stock closing prices. Each stock data served as the additional contributing factor to each other, hence when forecasting T-mobile stock closing price, Verizon stock closing prices data is the additional contributing factor, and vice versa. On Verizon data, the proposed approach achieved Relative RMSE of 2.168, while on T-mobile data it achieved Relative RMSE of 2.730, beating other approaches by achieving up to approximately $81 \%$ error score reduction.

\subsection{A Summary of the Reviews}

Numerous researches have been conducted on stock price forecasting utilizing many models, including LSTM, BLSTM, CNN, and CNN-LSTM. LSTM approaches show undeniable successes on time series processing, especially when combined with other approaches. Several researches on BLSTM proves that such approach performs similarly to LSTM without further combination of methods. However, BLSTM is found to achieve only slightly better results than LSTM. The problem behind this may come from the pattern of stock price data itself that BLSTM may not be able to understand better than LSTM, or it can also due to the optimizations used for BLSTM is not yet the best method.

CNN provides a unique way to process time series data and yields quite good results. CNN-LSTM provides a unique perspective of processing time series data, in which features are extracted using convolutional layers to later be used by 
LSTM layers for retaining past information for better forecasting capability with good results and promising for usage in real-life. However, never has any research proposed the investigation of how structure of the model affects performance of the model.

\section{EXPERIMENTS}

We evaluated LSTM, BLSTM, CNN, and CNN-LSTM on the BBRI.JK dataset from Yahoo Finance [36] from January $1^{\text {st }}$, 2012 to December $17^{\text {th }}, 2019$ with 1,990 entries. We divided the dataset into training and test set, with $80 \%$ for training set and $20 \%$ for test set. A preprocessing on the data was also done by normalizing the data to values between 0 and 1 . shows several values of BBRI.JK and plots the closing price of BBRI.JK.

Table 1: Sample Data from BBRI.JK Yahoo Finance Data

\begin{tabular}{|c|c|c|c|c|c|c|}
\hline Date & Open & High & Low & Close & $\begin{array}{c}\text { Adjusted } \\
\text { Close }\end{array}$ & Volume \\
\hline $\begin{array}{c}1 / 2 / 201 \\
2\end{array}$ & 1360 & 1370 & 1350 & 1360 & 543.916748 & 15835000 \\
\hline $\begin{array}{c}1 / 3 / 201 \\
2\end{array}$ & 1380 & 1390 & 1360 & 1380 & 551.915527 & 81980000 \\
\hline $\begin{array}{c}1 / 4 / 201 \\
2\end{array}$ & 1390 & 1390 & 1370 & 1390 & 555.914978 & 151180000 \\
\hline $\begin{array}{c}1 / 5 / 201 \\
2\end{array}$ & 1390 & 1400 & 1370 & 1390 & 555.914978 & 134337500 \\
\hline
\end{tabular}

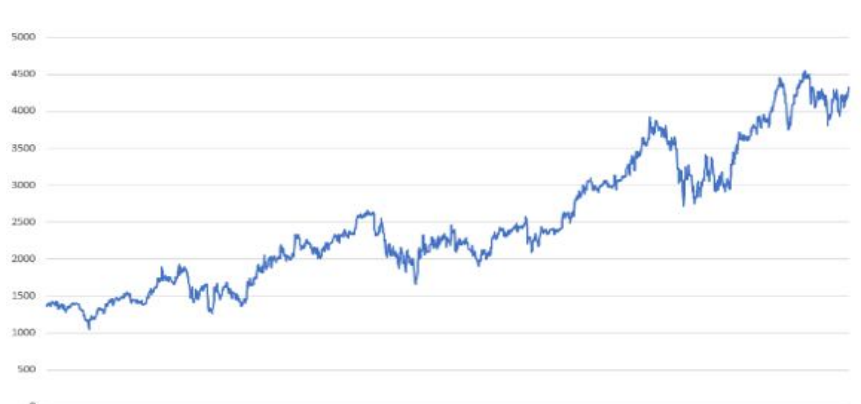

Figure 1: Plot of BBRI.JK Closing Price Data

The performance of each model is measured by RMSE. The variations of our experiments include the used optimizer, learning rate, batch size, and epoch. The used optimizers are Adam, RMSprop, and SGD with momentum (SGDM) with momentum value of 0.9. The learning rate values are 0.001 , 0.005 , and 0.01 . Our batch size also varies between 1 and 200, and the number of epochs from 1 to 10. Error! Reference source not found. shows our models' structures.

Table 2: Architecture of Experimented Models

\begin{tabular}{|c|l|}
\hline \multicolumn{1}{|c|}{ Model } & \multicolumn{1}{|c|}{ Layers } \\
\hline \multirow{3}{*}{ LSTM } & LSTM \\
\cline { 2 - 2 } & LSTM \\
\cline { 2 - 2 } & Fully Connected \\
\hline
\end{tabular}

\begin{tabular}{|l|l|}
\hline & Fully Connected \\
\hline \multirow{4}{*}{ BLSTM } & Bidirectional \\
\cline { 2 - 2 } & Bidirectional \\
\cline { 2 - 2 } & Fully Connected \\
\cline { 2 - 2 } & Fully Connected \\
\hline \multirow{4}{*}{ CNN } & Conv1D \\
\cline { 2 - 2 } & MaxPool1D \\
\cline { 2 - 2 } & Flatten \\
\cline { 2 - 2 } & Fully Connected \\
\hline \multirow{5}{*}{ CNN-LSTM } & Time Distributed (Conv1D) \\
\cline { 2 - 2 } & Time Distributed (MaxPool1D) \\
\cline { 2 - 2 } & Time Distributed (Flatten) \\
\cline { 2 - 2 } & LSTM \\
\cline { 2 - 2 } & Fully Connected \\
\hline
\end{tabular}

\section{RESULTS AND DISCUSSION}

After conducting the experiments, we found that CNN-LSTM provides the best result with RMSE score of 1.129830672 compared to other models. Full results of our experiments' best results for each model and the model's variations can be found on Error! Reference source not found. and plots of best and worst model's forecasts from Error! Reference source not found. are given in Error! Reference source not found.

CNN-LSTM provides the best result based on RMSE with only 1 epoch of training using SGDM and learning rate of 0.01 . This would be due to the good capability of both CNN for extracting features based on the normalized training data and LSTM layers to retain memory of past stock price value(s). In contrast, CNN gives the worst result of all experiments and that the best value is obtained after more training with large batch size. It can be concluded that CNN utilizes more resources as compared to other approaches but yields poor results. We believe that the reason of why CNN combined with LSTM outperforms CNN is the presence of LSTM layers after CNN, which helps retain past stock prices and forecasts better as opposed to only using fully connected layer after $\mathrm{CNN}$.

Table 3: Best Results for Each Model in Experiment

\begin{tabular}{|l|l|l|l|l|}
\hline \multicolumn{1}{|c|}{ Model } & \multicolumn{1}{c|}{ LSTM } & BLSTM & \multicolumn{1}{c|}{ CNN } & CNN-LSTM \\
\hline $\begin{array}{l}\text { Optimize } \\
\text { r }\end{array}$ & $\begin{array}{l}\text { RMSpro } \\
\text { p }\end{array}$ & SGDM & Adam & SGDM \\
\hline $\begin{array}{l}\text { Learning } \\
\text { Rate }\end{array}$ & 0.005 & 0.01 & 0.001 & 0.01 \\
\hline Epoch(s) & 1 & 1 & 10 & 1 \\
\hline $\begin{array}{l}\text { Batch } \\
\text { Size }\end{array}$ & 1 & 1 & 200 & 1 \\
\hline RMSE & 8.364372 & 5.12 & $\begin{array}{l}19.5257 \\
5\end{array}$ & $\mathbf{1 . 1 2 9 8 3 1}$ \\
\hline
\end{tabular}


(a)
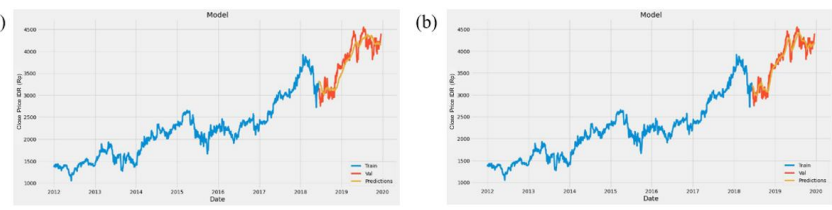

Figure 2: Forecasts by (a) CNN as the worst and (b) CNN-LSTM as the best performing model from Error! Reference source not found.. Forecasts are within the period of November $11^{\text {th }}, 2018$ until December $18^{\text {th }}, 2019$. The $\mathrm{x}$-axis denotes the year of the data while $\mathrm{y}$-axis denotes BBRI.JK stock's closing price per day in Indonesia Rupiah (IDR) currency. Training data, test data, and forecasting results are shown by blue, red, and orange curves respectively.

In line with the summary of the review on stock price forecasting, LSTM and BLSTM are competitive despite using different optimizer and learning rate value. It can also be observed that the difference in RMSE, despite being about 3.2 points, is quite large as opposed to what other related works on BLSTM reported when compared to LSTM. This may be because BLSTM has the capacity to understand context in forecasting, thus BLSTM has better understanding of the pattern of the stock prices compared to LSTM.

\section{CONCLUSION}

We provided a review of the progresses made for serial data processing in general, especially for time series processing. The review is extended with a more specific topic of stock price forecasting. Both reviews provide related works on each review's topic, and several datasets are also introduced. In addition, we also experimented on BBRI.JK dataset from Yahoo Finance of 1,990 entries and attained best RMSE score of approximately 1.13 with the utilization of CNN-LSTM, SGD with momentum of 0.9 , and learning rate of 0.01 . The best performing model from this paper's experiments is capable of forecasting stock prices with minimum error with univariate data of prior closing prices with few training resources and light architecture, therefore is suitable and should be capable of forecasting stock prices in times of catastrophic events or unpredictable times. Better forecasting can be obtained by further tuning the model with more recent stock price data.

Based on the reviews and our experiments on stock price forecasting, LSTM and BLSTM may be employed for stock price forecasting as it provides familiarity with general time series data processing and existing works show that good results are attained despite attaining competitive results with BLSTM achieving a better result. CNN is quite unique for time series processing as the forecasting is done by the fully connected layers, but the results are not as good as other approaches. As for CNN-LSTM, it has been proven to yield good results and it may be of great benefit to investigate how the modification of the structure of CNN-LSTM model, either increasing or decreasing number of convolutional layers, LSTM layers, and/or LSTM units, alters the performance of the model.

\section{REFERENCES}

1. Felsen J. Learning pattern recognition techniques applied to stock market forecasting. IEEE Transactions on Systems, Man, and Cybernetics. 1975;(6): p. 583-594.

2. Tawarish M, Satyanarayana K. A Review on Pricing Prediction on Stock Market by Different Techniques in the Field of Data Mining and Genetic Algorithm. International Journal of Advanced Trends in Computer Science and Engineering. 2019 February.

3. Suganya R, Kumar A S. Stock Price Prediction Using Tech News Based Soft Computing Approach. International Journal of Advanced Trends in Computer Science and Engineering. 2020 April.

4. Azubuike IM, Kosemoni OA. A Comparison of Univariate and Multivariate Time Series Approaches to Modeling Currency Exchange Rate. British Journal of Mathematics \& Computer Science. 2017; 21(4).

5. Iwok IA, Okpe AS. A Comparative Study between Univariate and Multivariate Linear Stationary Time Series Models. American Journal of Mathematics and Statistics. 2016; 6(5).

6. Brown MS, Pelosi M, Dirska H. Dynamic-radius Species-conserving Genetic Algorithm for. Machine Learning and Data Mining in Pattern. 2013;: p. 27-41.

7. Chen D, Sain SL, Guo K. Data mining for the online retail industry: A case study of RFM model-based customer segmentation using data mining. Journal of Database Marketing and Customer Strategy Management. 2012 August 27; 19(3): p. 197-208.

8. NRoS. National Records of Scotland. [Online].; 2019 [cited 2020 April 2. Available from: https://www.nrscotland.gov.uk/statistics-and-data/statist ics/statistics-by-theme/vital-events/births/births-time-ser ies-data.

9. Candanedo LM, Feldheim V. Accurate occupancy detection of an office room from light, temperature, humidity and $\mathrm{CO} 2$ measurements using statistical learning models. Energy and Buildings. 2016 January; 112(5): p. 28-39.

10. Andrzejak R, Lehnertz K, Rieke C, Mormann F, David P, Elger $C$. Indications of nonlinear deterministic and finite dimensional structures in time series of brain electrical activity: Dependence on recording region and brain state. Phys. Rev. 2001 December.

11. Dong E, Du H, Gardner L. An interactive web-based dashboard to track COVID-19 in real-time. The Lancet Infectious Diseases. 2020. 
12. UMLR. UCI Machine Learning Repository. [Online]. [cited 2020 April 3. Available from: https://archive.ics.uci.edu/ml/datasets.php?format=\&tas $\mathrm{k}=\&$ att $=\&$ area $=\&$ numAtt $=\&$ numIns $=\&$ type $=\mathrm{ts} \&$ sort $=\mathrm{ta}$ skUp\&view=table.

13. Kaggle. Kaggle. [Online]. [cited 2020 April 3. Available from: https://www.kaggle.com/tags/time-series.

14. ODD. Outlier Detection Datasets (ODDS). [Online]. [cited 2020 April 3. Available from: http://odds.cs.stonybrook.edu/.

15. Dau HA, Keogh E, Kamgar K, Yeh CCM, Zhu Y, Gharghabi S, et al. The UCR Time Series Classification Archive. 2019.

16. Elman JL. Finding Structure in Time. Cognitive Science. 1990; 14: p. 179-211.

17. Jordan MI. Serial order: A parallel distributed processing approach. Technical Report. San Diego: University of California, Institute for Cognitive Science; 1986.

18. Hochreiter S, Schmidhuber J. LONG SHORT-TERM MEMORY. Neural Computation. 1997; 9(8).

19. Graves A, Schmidhuber J. Framewise phoneme classification with bidirectional LSTM and other neural network architectures. Neural Networks. 2005 July; 18(5-6): p. 602-610.

20. Krizhevsky A, Sutskever I, Hinton GE. ImageNet Classification with Deep Convolutional Neural Networks. Advances in Neural Information Processing Systems. 2012; 25(2): p. 1106-1114.

21. Donahue J, Hendricks LA, Rohrbach M, Venugopalan S, Guadarrama S, Saenko K, et al. Long-term Recurrent Convolutional Networks for Visual Recognition and Description. IEEE Conference on Computer Vision and Pattern Recognition (CVPR). 2015 June.

22. Akbilgic O, Bozdogan H, Balaban ME. A novel Hybrid RBF Neural Networks model as a forecaster, Statistics and Computing. 2013.

23. Quotemedia. Quandl. [Online]. [cited 2020 April 3. Available from: https://www.quandl.com/data/EOD-End-of-Day-US-Sto ck-Prices.

24. Yahoo!. Yahoo Finance. [Online]. [cited 2020 April 3. Available from: https://finance.yahoo.com/.

25. Roondiwala M, Patel H, Varma S. Predicting Stock Prices Using LSTM. International Journal of Science and Research (IJSR). 2017 April; 6(4).

26. Sun S, Wei Y, Wang S. AdaBoost-LSTM Ensemble Learning for Financial Time Series Forecasting. International Conference on Computational Science. 2018 June.

27. Akita R, Yoshihara A, Matsubara T, Uehara K. Deep learning for stock prediction using numerical and textual information. IEEE/ACIS 15th International Conference on Computer and Information Science (ICIS). 2016 June.

28. Joo IT, Choi SH. Stock Prediction Model based on Bidirectional LSTM. Journal of the Korea Institute of
Information and Communication Technology. 2018 April; 11(2).

29. Jia M, Huang J, Pang L, Zhao Q. Analysis and Research on Stock Price of LSTM and Bidirectional LSTM Neural Network. Advances in Computer Science Research. 2019; 90.

30. Althelaya KA, El-Alfy ESM, Salahadin M. Evaluation of Bidirectional LSTM for Short- and Long-Term Stock Market Prediction. 9th International Conference on Information and Communication Systems (ICICS). 2018.

31. Gunduz $\mathrm{H}, \mathrm{YY}, \& \mathrm{CZ}$. Intraday prediction of Borsa Istanbul using convolutional neural networks and feature correlations. Knowledge-Based Systems. 2017; 137: p. 138-148.

32. Hoseinzade E, Haratizadeh S. CNNPred: CNN-based stock market prediction using several data sources. Expert Systems with Applications. 2019 March; 129: p. 273-285.

33. Chen S, He H. Stock Prediction Using Convolutional Neural Network. IOP Conference Series: Materials Science and Engineering. 2018; 435(1).

34. Li C, Zhang X, Qaosar M, Ahmed S, Alam KMR, Morimoto Y. Multi-factor Based Stock Price Prediction Using Hybrid Neural Networks with Attention Mechanism. IEEE Intl Conf on Dependable, Autonomic and Secure Computing, Intl Conf on Pervasive Intelligence and. 2019.

35. $\mathrm{Hu} \mathrm{C}, \mathrm{Hu}$ Y, Seo S. A Deep Structural Model for Analyzing Correlated Multivariate Time Series. 18th IEEE International Conference On Machine Learning And Applications (ICMLA). 2019 December.

36. Yahoo!. Yahoo Finance. [Online]. [cited 2020 April 2. Available from: https://finance.yahoo.com/quote/BBRI.JK/history?p=BB RI.JK. 